\title{
Adaptación de la Escala de Probabilidad de Perdón a población adolescente ARGENTINA
}

\author{
Adaptation of Forgiveness Likelihood Scale to Argentinian adolescent \\ POPULATION
}

\author{
María Soledad Menghi ${ }^{*}$, Lucas Marcelo Rodriguez ${ }^{*}$, María Candela Tortul ${ }^{* *}$ Y

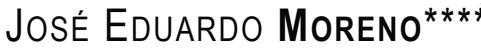

\begin{abstract}
*Doctora en Psicología. Becaria Posdoctoral del Consejo Nacional de Investigaciones Científicas y Técnicas (CONICET) y Docente en la Facultad Teresa de Ávila de la Universidad Católica Argentina (UCA).

E-Mail: msmenghi@uca.edu.ar

**Licenciado en Psicología. Becario Doctoral del Consejo Nacional de Investigaciones Científicas y Técnicas (CONICET) y Docente Adjunto de la Facultad Teresa de Ávila de la Universidad Católica Argentina (UCA).

E-Mail: lucasmarcelorodriguez@gmail.com

***Licenciada en Psicología. Becaria Doctoral del Consejo Nacional de Investigaciones Científicas y Técnicas (CONICET) y Docente Asistente de la Facultad Teresa de Ávila de la Universidad Católica Argentina (UCA).

${ }^{* * * *}$ Doctor en Psicología. Miembro de la Carrera del Investigador Científico (CONICET) y Coordinador del Centro de Investigación Interdisciplinar en Valores, Integración y Desarrollo Social en la Facultad Teresa de Ávila de la Universidad Católica Argentina (UCA).

Centro de Investigación Interdisciplinar en Valores, Integración y Desarrollo Social. Facultad Teresa de Ávila Universidad Católica Argentina (UCA).

Buenos Aires 239. (3100) Paraná, Provincia de Entre Ríos. República Argentina.

Grupo Vinculado al Centro Interdisciplinario de Investigaciones en Psicología Matemática y Experimental Dr. Horacio J.A. Rimoldi (CIIPME)

Consejo Nacional de Investigaciones Científicas y Técnicas (CONICET).

Ciudad Autónoma de Buenos Aires. República Argentina.
\end{abstract}

\section{REsUmen}

El objetivo de la investigación que se informa fue adaptar la Escala de Probabilidad de Perdón de Rye y colaboradores (2001) a una población adolescente argentina. A tal fin se realizaron dos estudios con residentes de la ciudad de Paraná (Argentina). El Estudio 1 tuvo como objetivo evaluar la validez factorial de la escala y con el Estudio 2 se puso a prueba el modelo de la escala surgido en el Estudio 1 a través de un análisis factorial confirmatorio. La muestra del Estudio 1 estuvo compuesta por 170 adolescentes (85 varones y 85 mujeres), con una media de edad igual a $15.60(D E=1.30)$ y la muestra del Estudio 2 estuvo compuesta por 294 adolescentes (131 varo- nes y 163 mujeres), con una media de edad igual a $15.60(D E=1.35)$. Para el Estudio 1 se realizó un análisis factorial exploratorio. Previamente se evaluó el índice de adecuación muestral de KaiserMeyer-Olkin $(\mathrm{KMO}=.78)$ y la prueba de esfericidad de Bartlett $\left(\chi^{2}=317 ; p=.000\right)$, los cuales indicaron la pertinencia para realizar el análisis factorial exploratorio. Finalmente resultó un único factor que explicaba el $32.79 \%$ de la variancia. En cuanto a la consistencia interna de la escala se obtuvo un índice Alpha de Cronbach igual a .76 para los diez ítems en la muestra de 170 adolescentes.

Teniendo en cuenta el Estudio 2, el análisis factorial confirmatorio mostró un buen ajuste del modelo propuesto, con los siguientes resultados: 
$\chi^{2}(32)=86.27 ; p=.000, \chi^{2} / g l=2.69, \mathrm{CFI}=.93$, GFI $=.94$, RMR $=.059$ y RMSEA $=.076 . \mathrm{La}$ adaptación del instrumento mostró buenas propiedades psicométricas de la escala para su uso en población argentina.

Palabras clave: Perdón; Adolescentes; Propiedades psicométricas; Escala; Agravio.

\section{ABSTRACT}

Forgiveness has positive implications for the emotional and social development. Several investigations have concluded that there is a strong negative correlation between forgiveness and the expression of anger and depression. It has been empirically shown that forgiveness has positive effects on mental health and psychophysical wellbeing. Through this capability the negative feelings of revenge and resentment are transformed into more positive feelings such as compassion and generosity, allowing to convert a negative and painful past into a positive one, thus strengthening the experiences through a reflective and peaceful look. In adolescence grievance situations are usually very frequent. Faced with these situations forgiveness is of great importance to restore the bonds between peers or family; essential bonds for the emotional support at this stage. This clearly shows the importance in the profound study of forgiveness as a variable that fosters psychological health in adolescence. This requires having instruments with good psychometric properties that can measure the ability to forgive in the Spanish - speaking population, more precisely the one from Argentina. The objective of this paper is to adapt Rye's Forgiveness Likelihood Scale (2001) to the Argentinian adolescent population. Two studies were conducted for this purpose. The aim of Study 1 was to evaluate the factorial validity of the scale. Study 2 aimed at testing the scale model emerged in Study 1 through a confirmatory factor analysis. The Study 1 sample consisted of 170 adolescents (85 males and 85 females) with a mean age of $15.60(S D=1.30)$. The Study 2 sample consisted of 294 adolescents (131 males and 163 females) with a mean age of $15.60(S D=1.35)$. Both samples were from Paraná City (Argentina). In both studies the samples were obtained in the same way. It was contacted secondary schools authorities to give them information about our research and to get their permission to carry it out. Then, parents' authorization was asked (informed consent). The samples were taken in groups of 20 to 30 adolescents approximately. During the administration at least two researchers were present. It was obtained the KMO sampling adequacy Index (.78) and the Bartlett Test of Sphericity $\left(\chi^{2}=317 ; p=.000\right)$, both of them indicate that it is appropriate to conduct the AFE. From the factor analysis the result is the existence of a single factor that explains $32.79 \%$ of the variance. To assess the internal consistency of the Forgiveness Likelihood Scale, the Cronbach Alpha was calculated, showing a coefficient of .76 for the ten items of the scale in the sample of 170 adolescents.

As for Study 2, the results of confirmatory factor analysis are: $\chi^{2}(32)=86.27 ; p=.000$, $\chi^{2} / g l=2.69, \mathrm{CFI}=.93$, GFI $=.94, \mathrm{RMR}=.059$, and RMSEA $=.076$. These results indicate a good fit of the proposed model. Thus, both studies convey the good adaptation and psychometric properties of the Forgiveness Likelihood Scale to Argentinian adolescent population. One of the limitations of the present research is the lack of convergent and discriminant validity studies. Both studies were conducted with the original version of the scale, obtaining a positive correlation with the Enright Forgiveness Inventory and other forgiveness measures. Further research should include this type of studies.

Key words: Forgiveness; Adolescence; Psychometric properties; Scale; Grievance.

\section{INTRODUCCIÓN}

El perdón tiene implicaciones positivas para el desarrollo emocional y social de las personas. Diversas investigaciones han concluido que existe una fuerte correlación negativa entre el perdón y la expresión de ira y depresión. Por otro lado, se ha encontrado una correlación positiva entre el perdón y las medidas de bienestar psicológico (Coyle \& 
Enrigth, 1997). El grado de daño y las consecuencias ocasionadas por el evento a perdonar estarán influidos por el grado de cercanía afectiva en la relación antes de la ofensa. Estudios llevados a cabo en diferentes culturas y contextos (Rique \& Enright, 1998; Subkoviak et al., 1995) han confirmado que las personas más cercanas son las que nos hieren más. Sin embargo, el grado y la cualidad del cariño en la relación entre víctima y ofensor antes de la ofensa es lo que puede motivar más hacia el perdón (Enright \& Rique, 2000).

Se ha demostrado empíricamente que la capacidad de perdonar tiene efectos positivos sobre la salud mental y el bienestar psicofísico. A través de esta capacidad se transforman los sentimientos negativos de venganza y resentimiento en sentimientos más positivos como la compasión y generosidad, permitiendo convertir un pasado negativo y doloroso en positivo, fortaleciendo así las vivencias del presente a través de una mirada reflexiva y en paz (Castro Solano, 2010).

Casullo (2006) considera al perdón como un constructo psicosocial mediado por una dimensión subjetiva intrapsíquica y otra interpersonal. Esta autora también lo conceptualiza como un proceso complejo, ya que en el mismo intervienen funciones cognitivas, afectivas, motivacionales, comportamentales, decisionales e interpersonales (Casullo, 2008).

"Perdonar implica un cambio interno y al mismo tiempo prosocial hacia una figura o situación percibida como transgresora, en el contexto de un vínculo interpersonal" (Casu1lo, 2006, p. 11).

En el marco del estudio sobre el perdón se han desarrollado diversas escalas de autoinforme para evaluar el constructo (Regalia \& Paleari, 2014). Esta multiplicidad de escalas se debe no sólo a las discrepancias en su definición sino también a los diferentes niveles de especificidad en los que se puede abordar esta variable (perdón a uno mismo, perdón a otro, perdón a la situación en sí, perdón según el tipo de ofensa, según la relación víctima-victimario, etc.) (Casullo, 2008; Regalia \& Paleari, 2014).
Uno de los problemas en la medición del perdón es poder lograr una definición operativa sobre lo que el mismo es. Es más fácil encontrar acuerdo sobre lo que no es el perdón que sobre lo que el mismo implica. Es así que algunos autores lo distinguen de la reconciliación, siendo posible perdonar sin restablecer el vínculo con la persona a quien se ha perdonado (Moreno \& Pereyra, 2000; Rye et al., 2001). De esta forma Rye y colaboradores (2001) conceptualizan el perdón como la respuesta hacia un ofensor que implica dejar de lado el afecto negativo (como por ejemplo, la hostilidad), cogniciones (como por ejemplo, pensar en la venganza) y conductas negativas (como las agresiones verbales), pudiendo incluirse respuestas positivas hacia el ofensor. Dicha conceptualización está muy relacionada con el perdón interpersonal planteado por Enright y Rique (2000) el cual implica las esferas afectiva, cognitiva y conductual del ser humano. Esta relación en las conceptualizaciones ha quedado de manifiesto en estudios empíricos (cf., Rye et al., 2001) al observarse la correlación que tienen los instrumentos creados con esta base teórica, como por ejemplo: la Escala de Probabilidad de Perdón (Rye et al., 2001) y el Inventario de Perdón de Enright (Enright \& Rique, 2000).

Estudios realizados con población urbana en Argentina han puesto de manifiesto que gran parte de los sujetos considera importante perdonar. La población más joven (adolescentes y jóvenes) considera importante perdonar para tener una segunda oportunidad frente a la equivocación, vinculándose también el perdón con la paz interior. Los mismos estudios han evidenciado que los hombres están motivados a perdonar para olvidar y seguir hacia adelante y mejorar así la convivencia social, mientras que las mujeres consideran que hay que perdonar para ser perdonados, expresando también que es un indicador de inteligencia (Casullo, 2006). Por su parte, estudios internacionales han mostrado que las mujeres presentan mayores niveles que los varones en el perdón a sí mismas y a otros, aunque no se observan diferencias según sexo en los niveles de perdón 
a diversas situaciones (Maganto \& Garaigordobil, 2010).

Otros estudios en adolescentes argentinos hallaron diferencias significativas entre el perdón interpersonal y el tiempo transcurrido, observándose menores promedios de perdón con el transcurso del tiempo. También se encontraron diferencias significativas entre el perdón interpersonal y la profundidad del daño, siendo menores los promedios de perdón cuando el daño es muy profundo (Rodriguez \& Moreno, 2013).

En la etapa adolescente las situaciones de agravio suelen ser muy frecuentes. Un estudio reciente en población adolescente observó que los mismos mencionan en gran medida a las agresiones en general (verbal, física, indirecta o directa) como la principal situación de agravio (Rodriguez \& Moreno, en prensa). El acoso escolar se ha incrementado en los últimos años en la Argentina y estudios recientes (Resett, Costa, Murata \& Falcone, 2015) describen la frecuencia y modo de agresión entre pares. Esto marca la importancia que adquiere perdonar ante estas situaciones de ofensa para poder restituir los vínculos entre pares o familiares, fuentes indispensables de contención afectiva en esta etapa (Resett, Rodriguez \& Moreno, 2013).

A partir de lo antedicho queda en evidencia la importancia que adquiere profundizar en el estudio del perdón como variable promotora de salud psicológica. Para ello es necesario contar con instrumentos con adecuadas propiedades psicométricas que puedan dar cuenta y medir la capacidad de perdonar en población hispanohablante y más precisamente en la de Argentina.

En esta línea se pueden mencionar instrumentos locales como el de Casullo (2005, cf., Casullo \& Fernández-Liporace, 2005) denominado CAPER; esta es una escala que evalúa la capacidad de perdonar. La misma consta de los siguientes factores: resignificación de errores y experiencias negativas, actitudes hacia el ofensor, control de situaciones y sentimientos / pensamientos negativos, creencias que determinan el perdonar, sentimientos hacia sí mismo y aceptación de situaciones o hechos negativos.
Existen diversos instrumentos para medir el constructo perdón, sobre todo en idiomas extranjeros, principalmente inglés. Este es el caso de The Forgiveness Scale (Rye, 1998; Rye et al., 2001), la cual es una escala diseñada para medir el perdón frente a un ofensor en particular y lo mide en los niveles afectivo, cognitivo y conductual, abarcando dos dominios: la ausencia de negatividad y la presencia de perdón.

Otro instrumento es el Transgression Related Interpersonal Motivations Inventory (TRIM - Bell et al., 2014; McCullough et al., 1998), esta es una escala conformada por dos dimensiones: una de venganza (compuesta por 5 ítems, e.g., "haré que él / ella paguen") y la otra de evitación (consta de 7 ítems, por ejemplo, "guardo tanta distancia entre nosotros como sea posible"). Se puede obtener una puntuación total de perdón si los ítems son cargados a la inversa.

También se puede mencionar la Heartland Forgiveness Scale (Escala de Perdón) de Yamhure-Thompson y Snyder (2003). La misma evalúa tres dimensiones: el perdón a uno mismo, el perdón a otros y el perdón a situaciones.

Por otro lado, Boonyarit, Chuawanlee, Macaskill y Supparerkchaisakul (2013) validaron en un grupo de enfermeras de Tailandia, la Workplace Forgiveness Scale, para medir el perdón en las relaciones laborales sobre un hecho específico.

Si bien existen algunos instrumentos para evaluar el perdón en idioma español, frente a la relevancia que adquiere el constructo por lo expresado anteriormente, es de gran importancia poder contar con nuevos instrumentos para la población hispanohablante que evidencien buenas propiedades psicométricas.

En función de los antecedentes mencionados se plantearon dos estudios: El Estudio 1 cuyo objetivo fue evaluar la validez factorial de la Escala de Probabilidad de Perdón de Rye y otros (2001) en una población argentina de la Provincia de Entre Ríos, y el propósito del Estudio 2 fue poner a prueba el modelo de esta escala surgido en el Estudio 1 a partir de un análisis factorial confirmatorio. 
La decisión de validar esta escala estuvo motivada principalmente porque indaga la probabilidad de perdón en diferentes escenarios y situaciones posibles de agravio, muy habituales en la vida.

\section{Metodología}

Se realizó un primer contacto con las autoridades de las instituciones educativas de nivel medio, para informar sobre la investigación y obtener la correspondiente autorización para administrar la prueba. Luego se solicitó la firma de padres o tutores del correspondiente consentimiento informado, el cual era requisito indispensable para participar de la investigación.

Para la selección de los participantes de ambas muestras se empleó un muestreo no probabilístico, de tipo intencional.

\section{MUESTRAS}

Las muestras para ambos estudios estuvieron integradas por alumnos de instituciones públicas y privadas que se encontraban cursando el nivel secundario en la ciudad Paraná (Provincia de Entre Ríos - Argentina).

En el Estudio 1 participaron 170 alumnos, de los cuales el $50 \%$ eran varones y el resto mujeres, de 13 a 19 años y la edad promedio fue de 15 años $(M=15.60 ; D E=1.30)$.

Para el Estudio 2, la muestra estuvo compuesta por 294 alumnos (131 varones y 163 mujeres) de 13 a 19 años y la mayoría tenía 15 años $(M=15.60 ; D E=1.35)$.

\section{INSTRUMENTO}

Para ambos estudios se utilizó una tradución de la Escala de Probabilidad de Perdón (Forgiveness Likelihood Scale), creada por Mark Rye (1998; Rye et al., 2001).

Se utilizaron las traducciones de tres traductores calificados, y posteriormente se realizó una traducción funcional del test. Luego, la adaptación fue revisada por exper- tos. Se tuvieron en cuenta los pasos propuestos por Hambleton y Patsula (1999) para la adaptación de pruebas.

Dicha escala mide la probabilidad de perdón en diferentes escenarios y situaciones posibles de agravio y cuenta con 10 situaciones en las que se produce una ofensa o agravio, como por ejemplo: infidelidad, engaño, robo.

En la situación hipotética planteada, la persona que responde la escala es agraviada y se le pregunta acerca de la probabilidad de perdonar a la persona que la ofendió o agravió. Ella debe responder con una escala tipo Likert de 5 opciones a saber: (1) Nada probable, (2) Poco probable, (3) Algo probable, (4) Bastante probable y (5) Totalmente probable.

\section{Procedimiento}

La administración se realizó con grupos de 20 a 30 adolescentes aproximadamente y con la presencia al menos de dos investigadores del equipo de investigación.

\section{Resultados}

\section{ESTUDIO 1}

El objetivo del Estudio 1 era evaluar la validez factorial y para ello se realizó un análisis factorial exploratorio (AFE) con el método de componentes principales. Previamente se evaluó el índice de adecuación muestral de Kaiser-Meyer-Olkin $(\mathrm{KMO}=.78)$ y la prueba de esfericidad de Bartlett $\left(\chi^{2}=317 ; p=.000\right)$, los cuales indicaron que era pertinente realizar el AFE. En la Tabla 1 se presenta la estructura factorial de la escala. Resultó un único factor que explicaba el $32.79 \%$ de la variancia.

Para evaluar la consistencia interna de la Escala de Probabilidad de Perdón se calculó el coeficiente Alpha de Cronbach, obteniéndose un valor igual a .76 para los 10 ítems en la muestra de 170 adolescentes. 


\section{ESTUDIO 2}

Para poner a prueba el modelo de la Escala de Probabilidad de Perdón, se realizó un análisis factorial confirmatorio con el método de estimación de parámetros por máxima verosimilitud en una muestra de 294 adolescentes.

Para considerar el ajuste del modelo, se tuvieron en cuenta el índice comparativo de ajuste (CFI) y el índice de bondad de ajuste (GFI). Se calcularon como medidas del error el Root Mean Square Residual (RMR) y el promedio de los residuales estandarizados al cuadrado (RMSEA). Luego de realizar un primer análisis factorial confirmatorio se utilizaron las sugerencias obtenidas a través de los índices de modificación, el cual sugería incorporar una covariancia entre los errores de los ítems 1 y 2,4 y 5 , y 7 y 8 .

Los resultados del modelo incluyendo las covariancias mencionadas fueron los siguientes: $\chi^{2}(32)=86.27 ; p=.000, \chi^{2} / g l=2.69$, $\mathrm{CFI}=.93$, GFI $=.94, \mathrm{RMR}=.059 \mathrm{y}$ RMSEA $=.076$. Dichos resultados indicaron un buen ajuste del modelo propuesto.

En la Figura 1 se presenta el modelo de medición y las cargas factoriales estandarizadas de la escala estudiada.

\section{DISCUSIÓN Y CONCLUSIONES}

El objetivo del Estudio 1 era evaluar la validez factorial de la Escala de Probabilidad de Perdón de Rye y colaboradores (2001) en una población argentina (Provincia de Entre Ríos). Tanto el índice de adecuación muestral KMO como la prueba de esfericidad de Bartlett indicaron que era pertinente realizar el AFE.

Los estudios de validez factorial resultaron satisfactorios. Se observó que de la estructura factorial de la escala resultó un único factor, que explica el $32.79 \%$ de la variancia, replicando el modelo original propuesto por los autores de la Escala.

En relación a la consistencia interna, en el modelo de Rye y colaboradores (2001) el índice de Alpha de Cronbach fue igual a .81 y en este estudio con adolescentes argentinos fue igual a .76. Dicho coeficiente puede considerarse satisfactorio, ya que valores de buena estimación de consistencia interna serían entre .70 y .80 (Kaplan \& Saccuzzo, 2006).

Con respecto al Estudio 2, el objetivo era poner a prueba el modelo de la Escala de Probabilidad de Perdón surgido en el Estudio 1, mediante el análisis factorial confirmatorio (AFC). Los resultados del AFC indicaron un buen ajuste del modelo propuesto, con índice de ajuste comparativo (CFI) e índice de bondad de ajuste (GFI) superiores a .9, indicando un ajuste satisfactorio entre el modelo teórico y los datos empíricos (Cupani, 2012). Asimismo un $\chi^{2} / g l$ inferior a 2 indica un buen ajuste del modelo. En este caso fue de 2.69; y si bien es más elevado de lo esperado, los demás indicadores de ajuste e índices de error permiten inferir un buen ajuste del modelo teórico propuesto a los datos empíricos.

De esta forma, a partir de la realización de ambos estudios se ha logrado una buena adaptación de la Escala de Probabilidad de Perdón a población adolescente argentina. Cabe destacar que dicha adaptación presenta buenas propiedades psicométricas.

Una de las limitaciones de la investigación realizada es que no se hicieron estudios de validez convergente o discriminante. Dichos estudios fueron realizados con la versión original de la escala, obteniéndose una correlación positiva con la Escala de Perdón de Enright y otras medidas de perdón (Rye et al., 2001). Posteriores investigaciones deberían incluir este tipo de estudios.

Futuras líneas de investigación deberían continuar evaluando las propiedades psicométricas del instrumento, así como su utilización en otras franjas etarias como la juventud y la adultez. También es importante seguir trabajando en el fortalecimiento de pruebas psicológicas válidas y confiables para la medición de constructos de la Psicología Positiva en población adolescente de nuestro medio. De igual modo, se considera que los datos revelados en este estudio resultan un aporte enriquecedor al demostrar la utilidad y funcionamiento de la escala en nuestro medio. 
TABLA 1

Estructura factorial de la Escala de Probabilidad de Perdón

\begin{tabular}{|c|c|}
\hline İtem & $\begin{array}{c}\text { Carga } \\
\text { factorial }\end{array}$ \\
\hline $\begin{array}{l}\text { 1.- Le contaste a un amigo / a algo sobre vos que considerás humillante, que te avergüenza. El / } \\
\text { ella te prometió mantenerlo en secreto y, sin embargo, no respetó su promesa y se lo contó a va- } \\
\text { rias personas. ¿Qué probabilidad hay de que perdones a tu amigo / a? } \\
\text { 2.- Un / a amigo / a difundió un rumor muy desagradable sobre vos que es mentira y que modifi- } \\
\text { có negativamente el trato que la gente tiene con vos. ¿Qué probabilidad hay de que perdones a } \\
\text { tu amigo / a? } \\
\text { 3.- Tu pareja cortó con vos dejándote herido / a y confundido / a, porque empezó a salir con uno / } \\
\text { a de tus mejores amigos / as. ¿Qué probabilidad hay de que perdones a tu pareja? } \\
\text { 4.- Uno de tus familiares te humilló delante de otras personas contando una historia tuya que no } \\
\text { querías que nadie se entere. ¿Qué probabilidad hay de que perdones a tu familiar? } \\
\text { 5.- Tu pareja tuvo una salida nocturna en la cual mantuvo una relación sexual ocasional. ¿Qué } \\
\text { probabilidad hay de que perdones a tu pareja? } \\
\text { 6.- Tu amigo / a estuvo hablando mal de vos a espaldas tuyas. Cuando lo encaras, él / ella lo nie- } \\
\text { ga, aunque vos sabes que miente. ¿Qué probabilidad hay de que perdones a tu amigo / a? } \\
\text { 7.- Un amigo / a te pide prestado algo muy valioso para vos y luego lo pierde. Tu amigo / a se } \\
\text { niega a reponerlo. ¿Qué probabilidad hay de que perdones a tu amigo / a? } \\
\text { 8.- Le contás a un / una conocido / a sobre un trabajo que te interesa obtener y lo estás intentan- } \\
\text { do. Sin decirte nada este / a conocido / a se postula y lo obtiene. ¿Qué probabilidad hay de que } \\
\text { perdones a este conocido? } \\
\text { 9.- Una persona desconocida entró a tu casa y robó una importante suma de dinero. ¿Qué pro- } \\
\text { babilidad hay de que perdones a esta persona? } \\
\text { 10.- Una persona te invitó formalmente a una fiesta y vos aceptaste. Sin embargo, esta persona } \\
\text { canceló el compromiso y concurrió a la fiesta con alguien que considera más atractivo / a. ¿Qué } \\
\text { probabilidad hay de que perdones a esa persona? }\end{array}$ & $\begin{array}{l}.720 \\
.649 \\
.428 \\
.605 \\
.623 \\
.593\end{array}$ \\
\hline$\%$ de variancia & 32.79 \\
\hline
\end{tabular}


FIGURA 1

MODELO DE MEDICIÓN Y CARGAS FACTORIALES ESTANDARIZADAS DE LA ESCALA DE PERDÓN

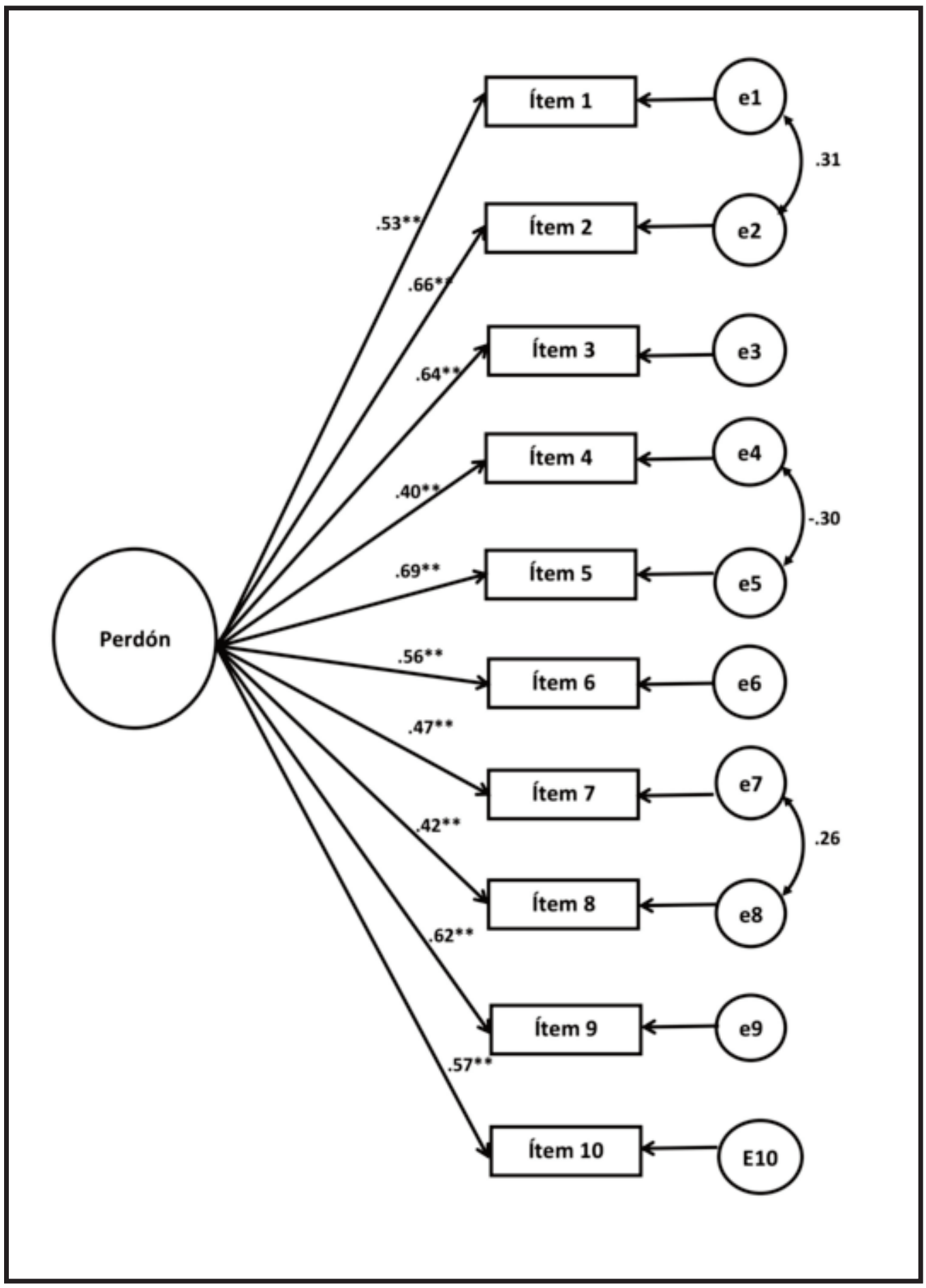




\section{REFERENCIAS BIBLIOGRÁFICAS}

Bell, C., Woodruff, E., Davis, D.E., Van Tongeren, D.R., Hook, J.N. \& Worthington, E.L. (2014). Community santification of forgiveness. Journal of Psychology \& Theology, 42(3), 243-251.

Boonyarit, I., Chuawanlee, W., Macaskill, A. \& Supparerkchaisakul, N. (2013). A psychometric analysis of the Workplace Forgiveness Scale. Europe's Journal of Psychology, 9(2), 319-338. http://doi.org/10.5964/ejop.v9i2.551

Castro Solano, A. (2010). Fundamentos de Psicología Positiva [Foundations of Positive Psychology]. Buenos Aires: Paidós.

Casullo, M. (2005). La capacidad para perdonar desde una perspectiva psicológica [The ability to forgive from a psychological perspective]. Revista de Psicología de la PUCP, 23(1), 3964.

Casullo, M.M. (2006). Las razones para perdonar: Concepciones populares o teorías implícitas [Reasons to forgive: Popular conceptions or implicit theories]. Psicodebate, 7, 9-20.

Casullo, M. (2008). Prácticas en Psicología Positiva [Practices in Positive Psychology]. Buenos Aires: Lugar Editorial.

Casullo, M. \& Fernández Liporace, M. (2005). Evaluación de la capacidad de perdonar. Desarrollo y validación de una escala [Assessment of the capacity to forgive. Development and validation of a scale]. Acta Psiquiátrica y Psicológica de América Latina, 51(1), 14-20.

Coyle, C.T. \& Enright, R.D. (1997). Forgiveness as an intervention goal with post-abortion men. Journal of Consulting and Clinical Psychology, 65, 1042-1046.

Cupani, M. (2012). Análisis de ecuaciones estructurales: Conceptos, etapas de desarrollo y un modelo de aplicación [Structural equation analysis: Concepts, developmental stages and an application model]. Revista Tesis, 1, 186-199.

Enright, R.D. \& Rique, J. (2000). The Enright Forgiveness Inventory. Manual, instrument and scoring guide. CA: Mind Garden.
Hambleton, R.K. \& Patsula, L. (1999). Increasing the validity of adapted tests: Myths to be avoided and guidelines for improving test adaptation practices. Journal of Applied Testing Technology, 1, 1-12.

Kaplan, R.M. \& Saccuzzo, D.P. (2006). Pruebas psicológicas: Principios, aplicaciones y temas [Psychological tests: Principles, applications and issues] (6ta. ed.). Madrid: International Thomson.

Maganto, C. \& Garaigordobil, M. (2010). Evaluación del perdón: Diferencias generacionales y diferencias por sexo [Assessment of forgiving: Generational and sex differences]. Revista Latinoamericana de Psicología, 42(3), 391-403.

McCullough, M.E., Rachal, K.C., Sandage, S.J., Worthington, E.L., Brown, S.W. \& Hight, T.L. (1998). Interpersonal forgiving in close relationships: II. Theoretical elaboration and measurement. Journal of Personality and Social Psychology, 75, 1586-1603.

Moreno, J.E. \& Pereyra, M. (2000). Cuestionario de Actitudes ante Situaciones de Agravio. Manual [Attitudes towards Offenders Questionnaire. Manual]. Libertador S. Martín, E. Ríos: Universidad Adventista del Plata (UAP).

Regalia, C. \& Paleari, F.G. (2014). Forgiveness of others scale. Encyclopedia of Quality of Life and Well-Being Research, 2338-2340. http:// doi.org/10.1007/978-94-007-0753-5_1077

Resett, S.A., Costa, D., Murata, C. \& Falcone, N. (2015). Equivalencia factorial del cuestionario de agresores / víctimas de Olweus según género [Factorial Invariance of the Olweus Bully / Bullied Questionnaire According to Gender]. Interdisciplinaria, 32(1), 169-181. https://doi. org/10.16888/interd.2015.32.1.9

Resett, S.A., Rodríguez, L.M. \& Moreno, J.E. (2013). Evaluación de la calidad de la amistad en niños argentinos [Evaluation of the quality of friendship in Argentinean children]. Acta Psiquiátrica y Psicológica de América Latina, 59 (2), 94-103. 
Rique, J. \& Enright, R.D. (Nov., 1998). Contexts of hurt and relation to forgiveness: An empirical analysis in four cultures. Trabajo presentado en el Meeting of the Association for Moral Education, Hanover, NH.

Rodriguez, L.M. \& Moreno, J.E. (2013). Profundidad del daño, tiempo transcurrido y perdón ante un agravio. Un estudio en adolescentes [Depth of damage, elapsed time, and forgiveness of a grievance. A study in adolescents]. Memorias del V Congreso Internacional de Investigación y Práctica Profesional en Psicología. Buenos Aires: Universidad de Buenos Aires.

Rodriguez, L.M. \& Moreno, J.E. (en prensa). Percepción de ofensas o agravios en adolescentes [Perception of offenses or grievances in adolescents]. Limite, Revista Interdisciplinaria de Filosofia y Psicología.

Rye, M.S. (1998). Evaluation of a secular and a religiously integrated forgiveness group therapy program for college students who have been wronged by a romantic partner. Tesis Doctoral no publicada. Bowling Green State University, Bowling Green, $\mathrm{OH}$.

Rye, M.S., Loiacono, D.M., Folck, C.D., Olszewski, B.T., Heim, T.A. \& Madia, B.P. (2001). Evaluation of the psychometric properties of two forgiveness scales. Current Psychology: Developmental, Learning, Personality, Social, 20, 260-277.

Subkoviak, M.J., Enright, R.D., Wu, C., Gassin, E.A., Freedman, S., Olson, L.M. \& Sarinopoulos, I. (1995). Measuring interpersonal forgiveness in late adolescence and middle adulthood. Journal of Adolescence, 18, 641-655.

Yamhure-Thompson, L. \& Snyder, C.R. (2003). Measuring forgiveness. En S.J. Lopez \& C.R. Snyder (Eds.), Positive psychological assessment: A handbook of models and measures. Washington: APA.

Centro de Investigación Interdisciplinar en Valores, Integración y Desarrollo Social Facultad Teresa de Ávila Universidad Católica Argentina (UCA) Paraná - Provincia de Entre Ríos Grupo Vinculado al Centro Interdisciplinario de Investigaciones en Psicología Matemática y Experimental Dr. Horacio J.A. Rimoldi (CIIPME) Consejo Nacional de Investigaciones Cientificas y Técnicas (CONICET) Ciudad Autónoma de Buenos Aires República Argentina

Fecha de recepción: 4 de octubre 2016 Fecha de aceptación: 23 de febrero de 2017 